\title{
Symmetry Exploitation of Diffraction Gratings to Enhance the Spectral Resolution
}

\author{
Eun Seong Lee* and Jae Yong Lee \\ Division of Convergence Technology, Korea Research Institute of Standards and Science, \\ Daejeon 305-340, Korea
}

(Received June 13, 2011 : revised August 11, 2011 : accepted August 23, 2011)

\begin{abstract}
A diffraction grating is a highly symmetric optical element with a physical structure that is invariant under translational spatial movements. The translational symmetry is reflected in the fields that are diffracted from the grating. Here, we introduce a plane-parallel mirror pair onto the grating, which translates the fields through double reflections, and we describe a method of exploiting the symmetry to enhance the spectral resolution of a diffraction grating beyond the limit that is set by the number of grooves. The mirror pair creates another virtual grating beside the original one, effectively doubling the number of grooves. Addition of more mirror pairs can further increase the effective number of grooves despite the increased complexity and difficulty of experimental implementation. We experimentally demonstrate the spectral linewidth reduction by a factor of four in a neon fluorescence spectrum. Even though the geometrical restriction on the mirror deployment limits our method to a certain range of the whole spectrum, as a practical application example, a bulky spectrometer that is nearly empty inside can be made compact without sacrificing the resolution.
\end{abstract}

Keywords: Diffraction grating, Translational symmetry, Spectral resolution, Spectrometer OCIS codes : (050.1950) Diffraction gratings; (300.6190) Spectrometers; (300.3700) Linewidth

\section{INTRODUCTION}

The diffraction grating consists of many grooves that are regularly spaced on a substrate surface. The spatial period of the groove lines typically ranges from one to several wavelengths of incident light. The wavefront of incident light falling on a grating surface is divided into many pieces, one at each groove line. The outgoing wavefront in a particular direction after the diffraction is composed of many interfering components from each groove line. Consequently, the direction in which all components are in phase is highly dependent on the wavelength of the incident light. In the years since its utility in the dispersion of multi-wavelength-component light was identified, it has been widely used for spectroscopy [1]. A tremendous number of spectroscopic applications are based upon grating-equipped spectrometers, including Raman scattering studies of molecular vibrational modes [2-4], fluorescent emission measurements of biological and chemical samples $[5,6]$, and optical spectrum analysis of photonics devices [7]. More recently, the diffraction grating has been found to be useful in the application of short laser pulse manipulation [8]. In this application, a multi-frequency-component short pulse laser beam is dispersed by a grating and focused on the Fourier plane, where each frequency component is controlled by a spatial light modulator to produce a variety of different shapes of laser pulses from the initial pulse after recombination through a second grating. This grating-based pulse manipulation has been widely adopted in chirped pulse amplifications to achieve high peak intensity laser beams $[9,10]$.

The spectral resolution of the diffraction grating is limited by the number of grooves that are covered by the incident beam [11], which is analogous to the diffraction-limited spatial resolution governed by the numerical aperture of the objective lens in optical imaging systems. Therefore, to achieve a high resolution, gratings are required to either be of a very large size or to have a high groove density. However, the high-precision ruling process can make this a difficult task. In this study, intrigued by the translational symmetry that is intrinsically imposed on the diffraction grating, we exploit the symmetry and present a very simple method to exhibit unprecedented spectral resolution with a limited groove number.

\footnotetext{
*Corresponding author: eslee@kriss.re.kr

Color versions of one or more of the figures in this paper are available online.
} 


\section{II . SYMMETRY OF DIFFRACTED FIELDS AND A PLANE-PARALLEL MIRROR PAIR AS A FIELD TRANSLATOR}

To exploit the intrinsic translational symmetry of diffraction gratings, we first want to have a close look into the field structure around a grating that is illuminated by a plane wave of wavelength $\lambda$. As the grating itself and the incident field are translationally invariant upon a $\mathrm{x}$-directed movement, as depicted in Fig. 1(a), the diffracted fields above the grating surface that consist of many diffraction orders have translational symmetries along the x-direction. Throughout the discussions following, we choose a transmission grating and scalar fields for the sake of convenience. Provided that the m-th order diffracted field $\phi_{m}(\vec{x})$ is a solution to the Helmholtz equation $\left(\nabla^{2}+k^{2}\right) \phi_{m}=0$ with appropriate boundary conditions, the field translated in space by $\vec{a}, \phi_{m}(\vec{x}-\vec{a})$ $\equiv \widehat{T} \vec{a} \phi_{m}(\vec{x})$, is also a solution because of the invariance of the equation under the translational operation; that is, $\left[\hat{T}_{\vec{a}}\right.$, $\left.\left(\nabla^{2}+k^{2}\right)\right] \phi_{m}(\vec{x})=0$. The plane wave solution $\phi(\vec{x})$ around the grating can be expressed below and above the grating as

$$
\begin{array}{ll}
\phi(\vec{x})=\phi_{i}\left(\vec{x} ; \vec{k}_{i}\right)=A\left(\vec{k}_{i}\right) \exp \left[-i \vec{k}_{i} \cdot \vec{x}\right] & \text { for } z \leq 0 \\
\phi(\vec{x})=\sum_{m} \phi_{m}\left(\vec{x} ; \vec{k}_{m}\right)=\sum_{m} A\left(\vec{k}_{m}\right) \exp \left[-i \vec{k}_{m} \cdot \vec{x}\right] & \text { for } z>0
\end{array}
$$

where $\vec{k}_{i}$ is the wavevector of the incident field below the grating, $\vec{k}_{m}$ is that of the m-th order diffracted field above the grating, and $\vec{k}_{i}^{2}=\vec{k}_{m}^{2}=\vec{k}^{2}$. If we apply the translation operator $\hat{T}_{\vec{a}}$ to the fields, then $\phi_{i}\left(\vec{x} ; \vec{k}_{i}\right)$ transforms to

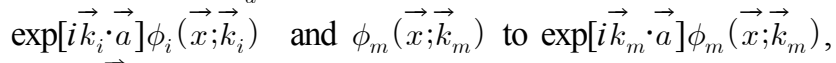
where $\vec{a}$ is a displacement vector. Generally, the field translation is accompanied by the phase shifts of wave functions. To realize the translational operation on the fields, we now consider an imaginary two-mirror element that has two large plane-parallel mirrors that are separated by a certain distance $d$ and reflect electromagnetic fields just twice: only once at each mirror, with no multiple reflections, as shown in Fig. 1(b).

Thus, any point in the fields is shifted in space by twice the mirror separation, $|\vec{a}|=2 d$, as indicated by the red arrow in the figure. Assuming that the phase of the field is not changed upon reflection, the mirror pair acts purely as a field translator. Eventually, we would like to identify a condition for the mirror separation and the slanting angle where both the diffracted original fields and the translated fields have the same phases. Then, the translated field will be exactly equivalent to the original field; that is, $\widehat{T}_{\vec{a}} \phi_{m}\left(\vec{x} ; \vec{k}_{m}\right)=\phi_{m}\left(\vec{x} ; \vec{k}_{m}\right)$ everywhere in the space. For this condition, we should satisfy the following relations from Fig. 1(b):

$$
\begin{aligned}
& \hat{T}_{\vec{a}} \phi_{m}\left(\vec{x} ; \vec{k}_{m}\right)=\exp \left[i \vec{k}_{m} \cdot \vec{a}\right] \phi_{m}\left(\vec{x} ; \vec{k}_{m}\right)=\phi_{m}\left(\vec{x} ; \vec{k}_{m}\right) \\
& \vec{k}_{m} \cdot \vec{a}=2 \pi n \text { or } 2 d \cos \left(\theta+\theta_{m}(\lambda)\right)=n \lambda \quad(n=\text { integer })
\end{aligned}
$$

where $\theta_{m}(\lambda)$ is the m-th diffraction angle, $d$ is the mirror

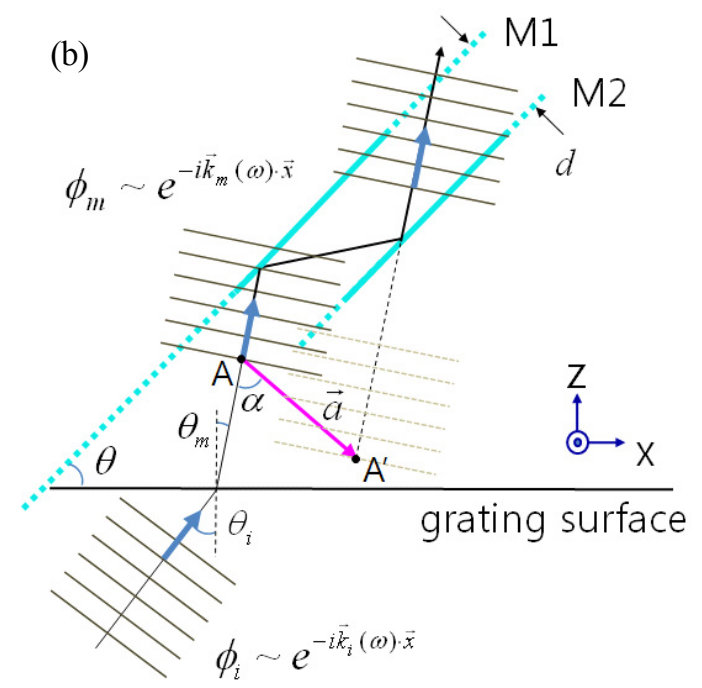

FIG. 1. Translation of diffracted fields by a pair of plane-parallel mirrors. (a) A grating and a few orders of diffracted fields from an incident plane wave. (b) The field translation that is induced by a pair of plane-parallel mirrors, M1 and M2. The diffracted fields from a grating are reflected twice by the mirrors, resulting in translated fields. The mirror pair acts as a field translator. The translation is represented by a vector $\vec{a}$. In a certain condition, the translated fields can be identical to the original diffracted fields due to the symmetry of the grating. 
separation, and $\theta$ is the slanting angle of the mirror pair. Simultaneously, we also require the fields to satisfy the grating equation, which is $b\left(\sin \theta_{m}-\sin \theta_{i}(\lambda)\right)=m \lambda$. Here, $b$ is the grating period and $\theta_{i}$ is the incident angle. Eq. (3) is the phase matching condition between the original and the translated fields. It is an identity relationship that should be satisfied for the arbitrary wavelength $\lambda$. Using the trigonometric identity $\sin (\theta-90)=-\cos \theta$, Eq. (3) can be expressed as $2 d \sin \left(\theta-90+\theta_{m}(\lambda)\right)=-n \lambda$. As the equation is satisfied simultaneously with the grating equation for every wavelength $\lambda$, we conclude that $\theta=90, \theta_{i}=0^{\circ}$, and $2 d m=$ $-n b$. As $n$ is an integer, the result implies that to fulfill the phase matching condition for all diffraction orders starting from the first order, the field translation $\vec{a}$ should be a horizontal shift (i.e., along the grating vector) by multiples of the grating period. This is what we can expect intuitively, and it is the same effect as would occur if the grating were translated by multiples of the grating period. The translational symmetry does not allow us to distinguish the original grating from the shifted grating.

\section{BEYOND THE LIMIT ON THE SPECTRAL RESOLUTION OF DIFFRACTION GRATINGS}

To exploit the symmetry nature of the fields that are formed around a grating system, we propose an optical configuration, as shown in Fig. 2(a), where a plane wave of wavelength $\lambda$ is incident on a grating plane and diffracted. We restrict the discussion to $m=-1$. The optical setup simply consists of one full mirror M2 and one half mirror M1 $(50 \%$ reflectivity) which are parallel to each other and separated by $d=n b / 2$, where $n$ is the number of grooves covered by the incident beam. The mirror planes are perpendicular to the grating plane and the angle of incidence is zero to fulfill the conditions obtained in the previous section. Half of the diffracted beam transmits through the half mirror. The other half is reflected toward the full mirror and is reflected again from the full mirror to become parallel to the transmitted beam. Consequently, the second half of the diffracted beam is the field translated by the mirror pair, which has the same phase and intensity as the first half. Therefore, the corresponding equivalent grating system can be depicted as shown in Fig. 2(b). The system works in a manner such that half of the original field and the other half of the field that is translated by $2 d$ interact to provide a larger aperture grating system. This system effectively doubles the number of grooves that are covered by the incident beam. The two-mirror configuration can be repeated once more by adding one more full-half mirror combination, and it consequently enhances the spectral resolution to quadruple that of the original diffraction grating. This concept is illustrated in Fig. 2(c), where the size of half mirror M3 is two times larger than that of mirror M1, and mirror M2 is extended to cover the reflected beam from M3. Additionally, the mirror separation between M2 and M3 is twice as large as that between M1 and M2. In principle, the spectral resolution can be enhanced indefinitely by repeating the full-half mirror combinations.

For an experimental demonstration of the enhanced spectral resolution, an approximately $7 \mathrm{~mm}$ long and $5 \mathrm{~mm}$ wide rectangular-shaped aperture was placed onto a large diffraction grating of 1000 grooves $/ \mathrm{mm}$ to simulate a grating of size $7 \mathrm{~mm} \times 5 \mathrm{~mm}$. The schematic of experimental setup is shown in Fig. 3. As a light source, a tunable diode laser operating around $773 \mathrm{~nm}$ with a linewidth of less than 300 $\mathrm{kHz}$ was coupled to a single mode fiber of NA0.12. The diverging laser beam from the output end of the fiber was collimated to make the beam size about $10 \mathrm{~mm}$ which was large enough to cover the entire area of the grating surface. The beam was made to strike the grating surface at a normal incidence, as in Fig. 2. Therefore, there were approxi- (a)

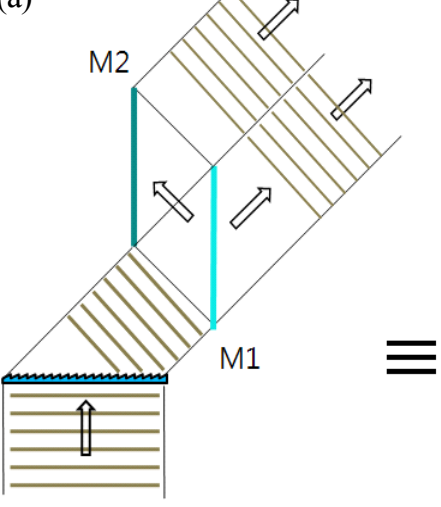

(b)

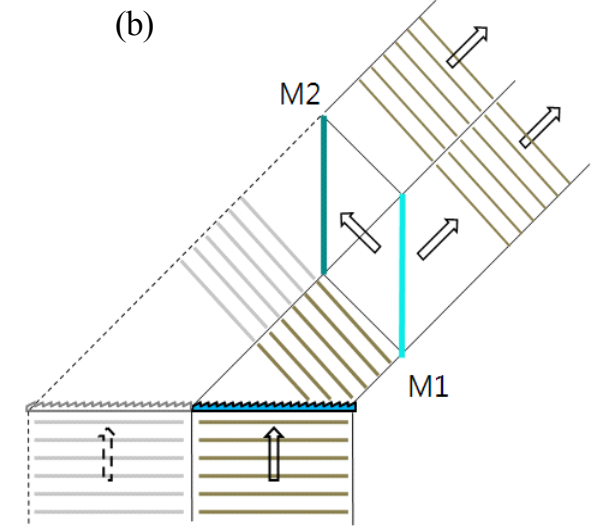

(c)

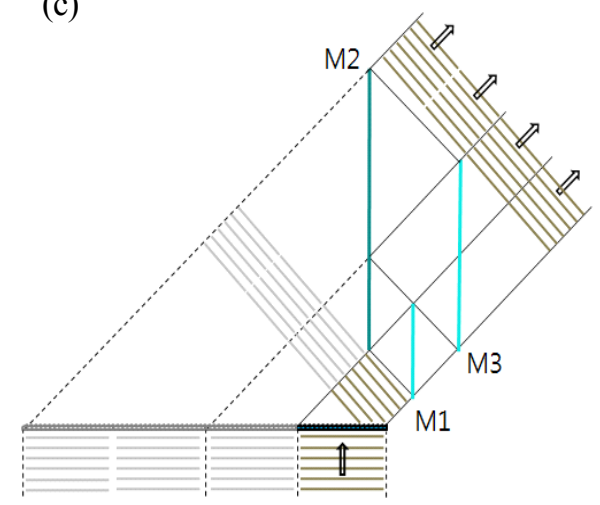

FIG. 2. Diffracted fields by a grating plus plane-parallel mirrors. (a) A pair of plane-parallel mirrors, the first of which is $50 \%$ reflective, doubles the width of the diffracted field. (b) The field passing through the mirror pair in (a) is equivalent to a diffracted field from a two times larger grating. (c) More mirror pairs can be added to increase the effective size of the grating or the number of grooves. The idea clearly shows that the spectral resolution of diffraction gratings can be enhanced as desired with a limited number of grooves. 


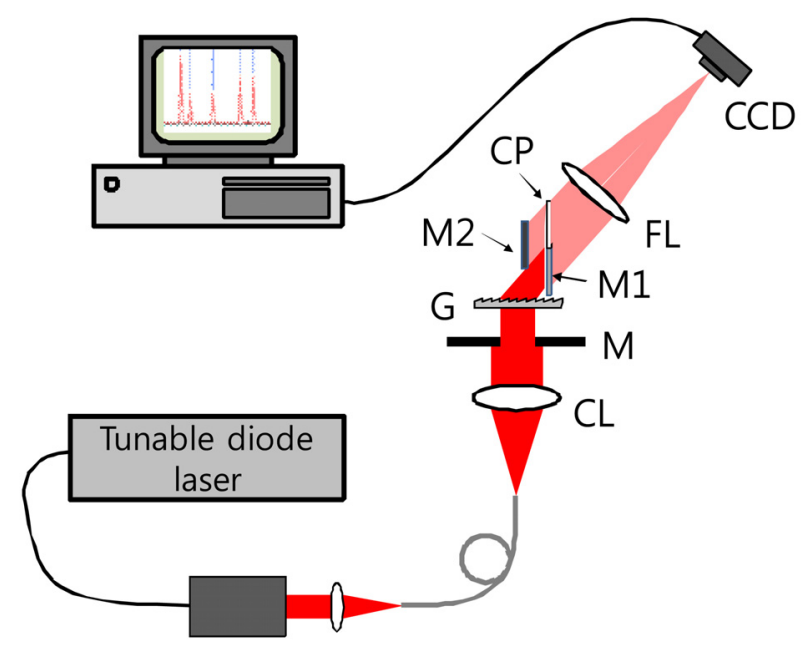

FIG. 3. Experimental setup. CL: collimation lens, M: rectangular mask, G: diffraction grating, M1: 50\% mirror, M2: $100 \%$ mirror, FL: focusing lens, CCD: charge coupled device, CP: compensating plate. The compensating plate is placed in the shifted beam path to compensate the dispersion induced by the half mirror. It also can be used for fine adjustment of the relative phase by slight tilting.

mately 7000 grooves that were covered by the laser beam, meaning that the first order spectral linewidth $\Delta \lambda$ would be $0.11 \mathrm{~nm}$ at $773 \mathrm{~nm}$ with the bare grating only. The first order diffracted beam at an angle of approximately $50.6^{\circ}$ to the grating normal was then focused onto a chargecoupled device (CCD) camera, which was at the focal plane of a plano-convex lens of focal length $250 \mathrm{~mm}$. As the laser wavelength scanned, the focal spot traversed the CCD image plane. The full width at half maximum (FWHM) of the spot corresponds to the spectral linewidth $\Delta \lambda$, and the enhancement of the spectral resolution is proven if the spectral linewidth is reduced in the entire tuning range of the diode laser. In Fig. 4, we present the spectral line profiles measured in the laser tuning range from $770 \mathrm{~nm}$ to $777.2 \mathrm{~nm}$. Fig. 4(a) is the result that was obtained with the bare grating only, and Fig. 4(b) is the result with the grating and a full-half mirror pair that is depicted in Fig. 2(a). The linewidths in Fig. 4(a) and 4(b) are approximately $0.11 \mathrm{~nm}$ and $0.06 \mathrm{~nm}$, respectively. These results agree well with our expectation. The spectral resolution of the mirrorsplus-grating system is thus twice that of the bare grating case. Here, we present only a limited number of laser lines, measured every $0.4 \mathrm{~nm}$ for graphical demonstrations. However, for the actual measurements, we performed the experiments in a continuous scanning manner.

In Fig. 4, we also present the neon emission spectra that were measured in our experiment. Due to the limited size of the CCD detection area, only two spectral lines out of many emission lines from a neon lamp are presented. One is positioned at $638.299 \mathrm{~nm}$, and the other brighter line is at $640.225 \mathrm{~nm}$. Fig. 4(c) corresponds to the neon spectrum that was obtained with the bare grating only, Fig. 4(d) to

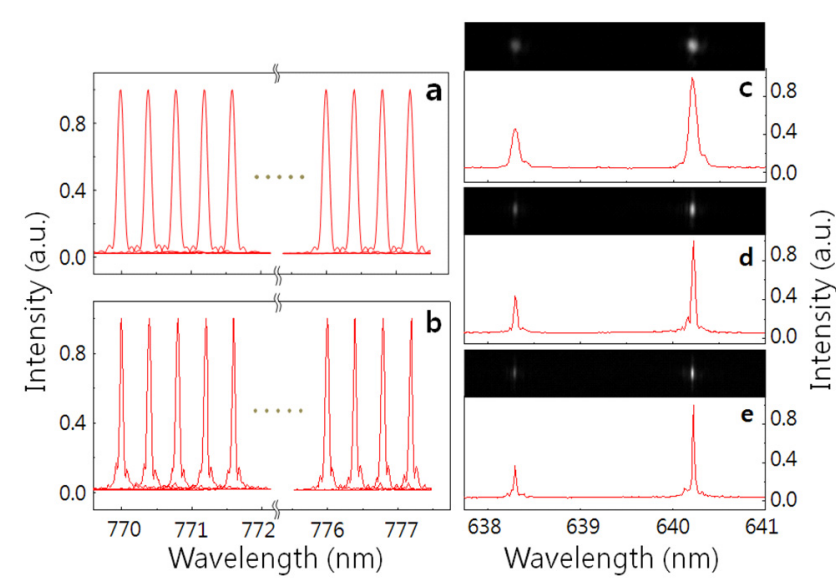

FIG. 4. Reduction of the spectral linewidth over a wide wavelength range. (a) The spectral line shapes of the diode laser that are measured while scanning its output wavelength. Here, the mirror pair is not added to the grating. (b) As the mirror pair is added, the line shapes become narrower throughout the entire tuning range. Each linewidth in (b) is half of that in (a). (c) The spectral line shapes of the neon fluorescent emission that is measured only with a grating. No mirror pair is added. (d) A mirror pair is added to the grating. (e) Two mirror pairs are added to the grating as shown in Fig. 2(c). Above each neon spectrum are the emission line spots that were imaged on the CCD detector. It should be noticed that along the dispersion direction the two emission spots become narrower as an additional mirror pair is added.

a single mirror pair with the grating, and Fig. 4(e) to double mirror pairs with the grating as depicted in Fig. 2(c). Since the relative phase between four diffracted beams is precisely adjusted, more careful alignment is necessary for the double mirror pair case. The spectral linewidths of the $640.225 \mathrm{~nm}$ emission for all three cases were measured as $0.095 \mathrm{~nm}, 0.049 \mathrm{~nm}$, and $0.025 \mathrm{~nm}$, respectively. Above each spectrum are the diffracted emission line spots that were imaged on the CCD detector. It should be noticed that along the dispersion direction the two emission spots become narrower as an additional mirror pair is added.

From the experimental results presented so far, we found that the proposed method works well for a fairly broad range of wavelength even though the working concept is depicted only for a single wavelength in Fig. 2. It can be attributed to the following reason. In the setup, we put the half mirror as close to the grating as possible, which is less than $15 \mathrm{~mm}$. Considering the dispersion of the grating $0.0015 \mathrm{rad} / \mathrm{nm}$ at $773 \mathrm{~nm}$, the swing angle for $7 \mathrm{~nm}$ wavelength change is just 0.0105 radians amounting to $0.16 \mathrm{~mm}$ transversal shift at the mirror. It is quite small compared to the diffracted beam width $4.6 \mathrm{~mm}$ and does not affect the spectral width so much. However, for a large change of wavelength, the mirror pair has to be moved vertically to be aligned for the new diffraction angle. Now we want to comment on the mirror misalignment effect on the phase mismatching. The most important part is to align 
the mirror pair at 90 degrees to the grating surface. If the angle is deviated from 90 degrees, then the relative phase of the two beams varies rapidly with wavelength. A simple calculation using Eq. (3) suggests that to keep the relative phase less than one quarter of $2 \pi$ from $770 \mathrm{~nm}$ to 777 $\mathrm{nm}$ the angular deviation $\Delta \theta$ should be less than 0.1 degree. The mirror alignment with this accuracy can be achieved by precision mechanical mounting systems without difficulty.

\section{OPERATION PRINCIPLE IN THE GEOMETRICAL OPTICS POINT OF VIEW}

Next, we want to look at the diffraction system from a different perspective in which the light field is represented by optical rays. This would allow us to see how the multiple two-mirror pairs in a grating system work from a geometrical optics point of view. Let's consider the interesting optical configuration depicted in Fig. 5, which exhibits scale symmetry. Thick lines represent full mirrors and thin lines represent half mirrors. Along the light propagation direction, the mirror pair scales double in mirror size and separation, and the number of rays is also doubled accordingly. At the smallest unit enlarged in a circle, the mirror pair splits a ray into two rays, between which the optical path difference is represented by $2 t \sin \theta_{m}$. At every step where the number of rays doubles, the optical path difference between two adjacent rays remains the same as the smallest unit. It is equivalent to a diffraction grating system, which has an optical path difference of $b \sin \theta_{m}$ between two adjacent grooves, where the horizontal separation $t$ of two mirrors corresponds to half of the grating period $b$. The equivalence is illustrated in Fig. 5. In ray optics, the two systems in the figure work in exactly the same manner; that is, the

(a)
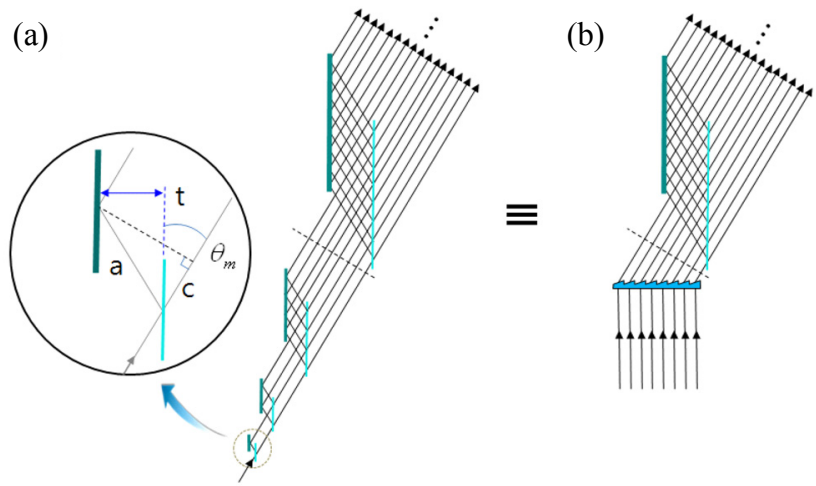

FIG. 5. Two-mirror operation from the geometrical optics point of view. Starting from a single unit of a full-half mirror pair in (a), the optical rays are doubled at each step of the two-mirror operation. Both multiple two-mirror operations in (a) and a grating in (b) produce equivalent diffracted fields at the plane that is indicated by the dashed lines. The in-phase conditions for all of the optical rays are exactly the same for both cases, and thus, so are the dispersions. diffraction angle of a specific wavelength $\lambda$ should satisfy the grating equation $2 t \sin \theta_{m}=b \sin \theta_{m}=m \lambda$. As the full-half mirror combinations can be added indefinitely in Fig. 5(a), the spectral resolution of the diffraction grating in Fig. 5(b) can also be enhanced as we wish. The scale symmetry of the optical configuration in Fig. 5(a) comes from Eq. (3), which implies that $\hat{T}_{l \vec{a}} \hat{T}_{(l-1) \bar{a}} \hat{T}_{(l-2) \bar{a}} \cdots \hat{T}_{\vec{a}} \phi_{m}\left(\vec{x} ; \vec{k}_{m}\right)=\hat{T}_{(l+1) \bar{a}} \hat{T}_{l a} \hat{T}_{(l-1) \bar{a}}$ $\cdots \hat{T}_{\vec{a}} \phi_{m}\left(\vec{x} ; \vec{k}_{m}\right)$, where $l$ is a positive integer. Although here we demonstrate this principle only for $2^{v}(v=$ positive integer) rays, it can be applied to any number of rays without difficulties. Therefore, any groove number can be considered by this framework.

For the case of non-zero incident angle upon a diffraction grating, we do not have solutions that satisfy Eq. (3) for the entire wavelength range. Instead, there is a small range, $\Delta \lambda$, around a specific wavelength $\lambda_{0}$ over which the equation is satisfied. This case would be important from a practical point of view because diffraction gratings in most spectrometers are generally used in non-zero incident angles. This case will be addressed in detail in future work.

\section{CONCLUSION}

In conclusion, exploiting the translational symmetry of the diffraction gratings, we have found a way to enhance the spectral resolution beyond the limit that is set by the number of grooves covered by an incident beam. A combination of full and half mirrors that are parallel to each other can optically replicate the original grating and place a virtual grating beside it, which effectively doubles the size of the grating, resulting in the resolution enhancement. This twomirror operation can be continued indefinitely for further enhancements by adding more full-half mirror combinations despite the increased complexity and difficulty of experimental implementation. Experimental demonstrations with a narrow band tunable diode laser and a neon fluorescence lamp were provided to show its promising utilities in spectroscopy applications. We have also presented an explanation of the two-mirror operation principle from the geometrical optics point of view, and an illustration with an interesting optical configuration that has scale symmetry was provided. Even though there are some drawbacks of our method such as the limitation of the working spectral range, it can be easily applied to any grating-based spectrometer due to its simplicity. As an example, bulky spectrometer systems in which most internal parts are simply empty space can be made smaller and more compact without degrading the spectral resolution. Since the present study can be applied only to the zero-incident angle, the future study will include the oblique incident angle case for more practical applications.

\section{ACKNOWLEDGMENT}

This work was supported by the grant from the Bio-signal 
Analysis Technology Innovation Program of the Ministry of Education, Science, and Technology, Republic of Korea.

\section{REFERENCES}

1. N. Savage, "Spectrometer," Nature Photon. 3, 601-602 (2009).

2. K. Kneipp, Y. Wang, H. Kneipp, L. T. Perelman, I. Itzkan, R. R. Dasari, and M. S. Feld, "Single molecule detection using surface-enhanced Raman scattering," Phys. Rev. Lett. 78, 1667-1670 (1997).

3. S. Nie and S. R. Emory, "Probing single molecules and single nanoparticles by surface-enhanced Raman scattering," Science 275, 1102-1106 (1997).

4. F. D. Angelis, G. Das, P. Candeloro, M. Patrini, M. Galli, A. Bek, M. Lazzarino, I. Maksymov, C. Liberale, L. C. Andreani, and E. D. Fabrizio, "Nanoscale chemical mapping using three-dimensional adiabatic compression of surface plasmon polaritons," Nature Nanotech. 5, 67-72 (2010).
5. S. Weiss, "Fluorescence spectroscopy of single biomolecules," Science 283, 1676-1683 (1999).

6. I. L. Medintz, A. R. Clapp, H. Mattoussi, E. R. Goldman, B. Fisher, and J. M. Mauro, "Self-assembled nanoscale biosensors based on quantum dot FRET donors," Nature Mater. 2, 630-638 (2003).

7. T. W. Ebbesen, H. J. Lezec, H. F. Ghaemi, T. Thio, and P. A. Wolff, "Extraordinary optical transmission through sub-wavelength hole arrays," Nature 391, 667-669 (1998).

8. N. Dudovich, D. Oron, and Y. Silberberg, "Single-pulse coherently controlled nonlinear Raman spectroscopy and microscopy," Nature 418, 512-514 (2002).

9. D. Yelin, D. Meshulach, and Y. Silberberg, "Adaptive femtosecond pulse compression," Opt. Lett. 22, 1793-1795 (1997).

10. K. Yamane, Z. Zhang, K. Oka, R. Morita, M. Yamashita, and A. Suguro, "Optical pulse compression to $3.4 \mathrm{fs}$ in the monocycle region by feedback phase compensation," Opt. Lett. 28, 2258-2260 (2003).

11. E. Hecht, Optics, 4th ed. (Addison Wesley Longman, Reading, MA, USA, 2002). 